Voix et Images

voixetimages

\title{
Un sous-genre hybride : la nouvelle romanesque
}

\section{Michel Biron}

Volume 30, numéro 1 (88), automne 2004

Le pseudonyme au Québec

URI : https://id.erudit.org/iderudit/009894ar

DOI : https://doi.org/10.7202/009894ar

Aller au sommaire du numéro

\section{Éditeur(s)}

Université du Québec à Montréal

\section{ISSN}

0318-9201 (imprimé)

1705-933X (numérique)

Découvrir la revue

Citer ce compte rendu

Biron, M. (2004). Compte rendu de [Un sous-genre hybride : la nouvelle

romanesque]. Voix et Images, 30(1), 125-130. https://doi.org/10.7202/009894ar d'utilisation que vous pouvez consulter en ligne.

https://apropos.erudit.org/fr/usagers/politique-dutilisation/ 


\section{R O M A N}

Un sous-genre hybride: la nouvelle romanesque

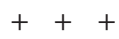

MICHEL BIRON

Université McGill

On trouve de plus en plus souvent de recueils de nouvelles construits autour de personnages et de scènes qui font retour d'un texte à l'autre. L'effet d'unité est accru de sorte que le caractère soi-disant fragmentaire ou hétérogène du traditionnel recueil de nouvelles ne correspond plus à ce qu'on lit. Au contraire, la convergence des univers juxtaposés devient si évidente qu'on a l'impression de se trouver devant un livre-concept; chaque nouvelle s'intègre fortement dans les autres et, à la limite, chacune se lit comme un chapitre d'un roman légèrement postmoderne. Ce sous-genre hybride pourrait s'appeler «la nouvelle romanesque». Il a l'avantage de ne pas exiger une architecture complexe comme celle du roman, tout en annulant l'aspect composite du recueil de nouvelles. Le lecteur n'a plus le sentiment de recommencer à zéro d'une nouvelle à l'autre, mais se trouve devant des tableaux qui s'imbriquent les uns dans les autres, quoique de façon moins organique et plus aléatoire que des chapitres romanesques. Un tel travail sur la forme permet au lecteur de découvrir des liens là où il n'en attendait pas. L'an dernier, par exemple, Lise Tremblay avait écrit une nouvelle romanesque avec La héronnière ${ }^{1}$. Cette année, deux nouvellistes publient de tels livres: ce sont Suzanne Myre avec $\mathrm{Hu}-$ mains aigres-doux ${ }^{2}$, qui est son deuxième recueil, et Michèle Péloquin, avec Les yeux des autres ${ }^{3}$, son premier livre. On verra aussi que le dernier recueil de Gilles Pellerin, $\ddot{I}$ (i tréma $)^{4}$, sans avoir recours au même procédé, vise aussi à dépasser le traditionnel recueil de nouvelles pour créer un objet unifié.

Le cheveu est essentiel pour les personnages de Humains aigres-doux, paru aux Éditions Marchand de feuilles qui décrivent Suzanne Myre comme «la nouvelliste la plus décapante de la nouvelle génération d'auteurs québécois». Symbole de la

$$
+++
$$

1 Lise Tremblay, La héronnière, Montréal, Leméac, 2003, 111 p. 2 Suzanne Myre, Humains aigres-doux, Montréal, Éditions Marchand de feuilles, 2004, 159 p. 3 Michèle Péloquin, Les yeux des autres, Montréal, XYZ éditeur, 2004, 134 p. 4 Gilles Pellerin, İ (i tréma), Québec, L'Instant même, 2004, 155 p. 
vanité humaine, la coiffure constitue la métaphore filée d'un bout à l'autre du recueil. L'une a une coiffure en forme de tour de Pise, l'autre en forme de tour Eiffel, et le coiffeur bisexuel Walter (prononcez «Voualtèr») est le grand héros de cette faune dont la vie tient à un cheveu. Dans «Au poil près», il fait une coupe à la Brad Pitt à un client avant de l'emmener chez lui pour le «croquer», puis l'assassiner tout bêtement. Il lui coupe aussi les deux oreilles, comme on le fait dans une corrida pour récompenser le toréador, afin de les ajouter à sa collection morbide. Walter revient dans presque toutes les nouvelles du recueil comme si le livre entier se déroulait autour de ce salon de coiffure (appelé «Épi en tête») situé sur la rue «Moron-Royal». C'est moins une unité de lieu qu'une unité de discours, c'est là que convergent toutes les rumeurs sociales, les mauvaises langues, les références moqueuses ou admiratives aux vedettes (la narratrice prend un malin plaisir à parler d'une coiffure qui serait un mix de Cruella et de Marie Laberge). Suzanne Myre observe avec humour ceux et celles qui connaissent Walter et les mille et un noms de ses teintures («bois franc laqué», "brisures d'automne», etc.). Ce sont des obsédés du paraître.

La première nouvelle, qui fait près de quarante pages, évoque une soirée «sushis» entre trois femmes. La narratrice ne sait pas trop pourquoi elle a accepté l'invitation, d'autant moins qu'elle "exècre» les sushis. Mais elle se retrouve devant l'amie de son amie, la championne des sushis. Commence entre elles la guerre des pointes assassines, des mots blessants, des allusions perfides, des questions indiscrètes: «Et toi, tes orgasmes, Sylvia, ils sont comment?» (33) Après le temps des hostilités, celui des confidences paraît tout aussi creux. La narratrice n'entre pas dans le jeu et finit par s'en aller brusquement. Elle traverse la rue et entre chez le voisin où se tient une bruyante fête. Elle y voit une BMW égratignée, dont l'histoire se trouvera dans la nouvelle «Une ambiance d'enfer». Ce n'est pas la seule allusion aux nouvelles qui suivent (et même à celles qui précèdent puisque la narratrice affirme avoir lu le premier livre de nouvelles de Suzanne Myre). Il est aussi question d'une femme qui déchire une lettre derrière un poteau, scène qui sera racontée dans «Maris, amants et femmes» (mais en plus tordu, puisque la femme découvre que ce n'est pas seulement son mari qui la trompe avec sa sœur, son amant fait de même). Bref, la nouvelle d'ouverture contient quantité d'allusions décousues qui trouvent cependant leur sens à la lecture des autres nouvelles du recueil.

Humains aigre-doux est une charge contre la société hypersuperficielle d'aujourd'hui. Nous avons droit à une reconstruction réussie du babil de ceux et celles qui se coiffent comme des vedettes, se croient pleins d'esprit et sont sûrs de vivre une vie parfaite et surtout enviable. La plupart sont jeunes, même si déjà le malheur de vieillir semble les préoccuper: ici, ce sont les seins qui tombent, là une calvitie précoce. Mais ils ne connaissent pas de grand malheur, outre les petits problèmes de coiffure. Ils ont la répartie facile et les dialogues, particulièrement bien frappés, font sourire. L'écriture de Suzanne Myre arrive aisément à nous faire croire à ce monde "aigre-doux", tantôt méchant tantôt sucré. Mais le problème, quand on dénonce la superficie, c'est qu'on ne peut s'empêcher de se croire supérieur aux personnages ainsi décrits. Dans «Naissance et mort d'une calvitie», la sœur du narrateur, Chrystelle, la maniaque aux sushis qu'on a déjà rencontrée dans la 
nouvelle du début, convainc son frère d'aller voir Walter, le maître-coiffeur. Chrystelle n'est pas un personnage sympathique; elle représente même tout ce que la narratrice déteste: «banalité, clichés, fausse féminité » (39). Mais le recueil s'attache à elle comme à un bouc-émissaire. Son frère - plus sympathique, celuilà - a une formule géniale pour la décrire: elle a «le don de ne jamais aller trop en profondeur» (129). L'expression ironique est évidemment péjorative dans le contexte de ces nouvelles. Elle pourrait s'appliquer à l'ensemble des personnages qui fréquentent le salon de Walter, c'est-à-dire à presque tous les personnages du recueil.

Des individus comme Chrystelle sont forcément artificiels, tournés vers leur extériorité et quelque peu ridicules. Les mépriser est une partie de plaisir; nous aimons croire que nous ne sommes pas aussi bêtes qu'eux. Se passionner pour les sushis, se faire teindre les cheveux couleur «Délire décadent», construire un cabanon pour améliorer sa vie de couple, ce n'est pas ainsi que nous, êtres évolués, choisissons de remplir le vide de l'existence. Soit. Mais quelque chose retient le lecteur d'adhérer entièrement à un tel jeu de massacre. On voudrait leur trouver quelque chose d'aimable, on voudrait ne pas simplement se contenter de les voir de loin, en passant. Mais Suzanne Myre ne nous donne pas beaucoup de chance de le faire, comme si elle avait pris le parti d'en rire. Ce n'est pas désagréable, mais l'ironie à répétition laisse un drôle de rictus à la fin.

Les yeux des autres de Michèle Péloquin contient, lui aussi, quelques personnages qui reviennent d'un texte à l'autre, mais ils sont beaucoup moins nombreux que dans le recueil de Suzanne Myre. Il y a d'abord la figure de l'enfant Bilou, symbole d'innocence et de confiance. Il a moins d'un an dans «Noix de cajou et gelée de canneberge», un peu plus dans «Un ciel bleu septembre», cinq ans dans «Avant la lettre», sept ou huit ans dans «La photo des vacances». Mais c'est la figure de la narratrice qui semble donner son unité à l'ensemble du recueil. On découvre sa vie peu à peu, à travers le regard des autres, mais aussi à travers ce qu'elle imagine qu'ils voient d'elle.

Dans la nouvelle d'ouverture, «Un ruban de satin jaune», la narratrice glisse cette petite phrase: «[...] ma vie finalement n'était pas un si grand désastre.» (14) Elle est assise sur l'herbe lors d'un repas en famille et se laisse doucement enivrer par le vin et par la musique du vent dans les feuilles d'un tilleul. Elle insiste sur la douceur de l'air, la beauté du moment, l'harmonie des lieux. Mais la petite phrase qui parle de désastre n'annonce rien de bon, même si, pour une fois, le désastre semble supportable. La formule suggère tout au plus une vision provisoirement moins sombre, comme si la narratrice goûtait les joies d'une rémission. Aussi le lecteur est-il à peine surpris quand la catastrophe se produit, redonnant à la vie son allure de "grand désastre». La mère, qui s'était allongée sous le tilleul, est morte. Sans raison apparente, elle s'est éteinte au milieu de sa sieste. La narratrice ne s'intéresse d'ailleurs pas aux raisons du drame et ne cherche pas à le lier à d'autres événements. Seul compte le souvenir très précis de cette scène au cours de laquelle 
elle a perdu sa mère. Ce ne sont que des notes visuelles ou sonores, des sensations qui restent longtemps et qui s'imprègnent dans la mémoire du corps. Des mots entendus au plus fort du drame, comme lorsque son frère jette à la poubelle le reste de la tarte aux fruits «en disant que, de toute façon, elle n'était pas mangeable. Oui, il a dit ça. Je me souviens de tout. D'absolument tout» (16-17). Jusqu'à un ruban de satin jaune oublié sur le bord de la terrasse et qu'elle met dans sa poche avant de quitter les lieux du drame.

Les événements importent généralement assez peu dans les nouvelles de Michèle Péloquin. Ils sont presque toujours attendus, même dans le cas de cette première nouvelle où l'on devine que la vie donnera tôt ou tard raison à celle qui l'associe à un "grand désastre». Les surprises sont rares, l'intrigue paraît secondaire et les chutes contiennent presque toujours une phrase de trop, comme si l'auteure ne savait pas comment conclure et voulait absolument que l'on comprenne le sens de la nouvelle («Enfin, j'ai pu pleurer», lit-on à la fin de la première nouvelle). Mais l'intérêt de ces nouvelles est ailleurs que dans le récit lui-même. Il vient de la précision du trait et du sentiment de proximité, voire de solidarité que l'on éprouve à l'égard de chacun des personnages et qui atténue le "grand désastre» même si on ne parvient jamais à l'oublier tout à fait. Ce sont des scènes souvent tirées de la vie quotidienne, arrachées à la banalité. Dans ce premier livre, Michèle Péloquin parvient à traduire avec simplicité les aléas de la vie contemporaine, entre les blessures de l'amour, la famille décomposée, le travail incertain et l'immense solitude de chaque individu.

L'amour constitue sans doute le plus important thème du recueil. Il surgit un peu partout, même dans les nouvelles qui parlent d'autre chose. La narratrice a de curieuses formules, comme celle-ci dans «Noix de cajou et gelée de canneberge»: «Je repense à toutes les fois où j'ai dit "je t'aime" au lieu de "aime-moi".» (20) Dans chaque cas, on dirait que l'amour est unidirectionnel et sans avenir. L'amour, sans cesse déçu, se négocie au rabais. La narratrice d'«Intermittence» se console par exemple en pensant aux plaisirs furtifs éprouvés quand son amant vient chez elle, résolue à se contenter de bien peu: «[...] des miettes d'amour valent mieux que pas d'amour du tout.» (41) L'amitié, souvent comparée à l'amour, ne vaut guère mieux. Elle naît au hasard d'un voyage ("Athènes-Amsterdam»), puis s'éteint dès le retour au pays. Toute forme de relation humaine paraît fragile, vouée à l'échec. Pourtant, Les yeux des autres n'est pas un livre sombre au bout du compte, en dépit des défaites amoureuses et de la solitude des êtres. Il y a d'abord la présence de l'enfant «Bilou», comme on l'a vu. Il s'agrippe à la narratrice, lui serre la main avec une telle force qu'elle se sent de nouveau présente au monde, avec une confiance renouvelée. Mais il y a plus que cet enfant qui suscite la sensation de vivre et qui autorise une forme de sérénité malgré le "grand désastre». Il y a aussi des instants magiques de félicité ou encore des moments si intenses que le corps semble perdre toute lourdeur pour épouser le paysage. Ces instants sont souvent associés à une couleur, comme dans «Orangé» ou "Autoroute 20, 6 h 17 »: «On dit que les gens heureux n'ont pas d'histoire. Ça n'est pas vrai. Moi, en ce moment, je suis le mauve de la lumière devenue rose, je suis le grain du ciel, la route que je sens défiler sous mes pieds.» (109) Ce refus d'exclure le bonheur de cet univers fictif qu'on appelle la littérature 
paraît assez caractéristique de la manière d'écrire de Michèle Péloquin. Sans naïveté, avec même une bonne dose d'ironie à l'égard des clichés contemporains (voir «Politically correct»), elle se hasarde à décrire ces moments furtifs où l'on a le sentiment d'exister pleinement.

Gilles Pellerin a fait de la nouvelle son territoire d'expérimentation privilégié. Éditeur, professeur, conférencier, il a écrit plusieurs recueils de nouvelles et a même publié une anthologie et un essai consacrés à ce "petit» genre ${ }^{5}$. Il vient de rééditer Ni le lieu ni l'heure paru d'abord en 1987, et de faire paraître un nouveau recueil au titre étrange, İ (i tréma). Contrairement aux livres de Suzanne Myre et de Michèle Péloquin, il ne s'agit pas de nouvelles dans lesquelles des personnages font retour. Mais il s'agit d'un recueil dont on aperçoit l'architecture générale avant même de commencer la lecture.

$\ddot{I}$ (i tréma) est un recueil de nouvelles très brèves, d'une ou deux pages tout au plus, quelquefois seulement d'une ou deux lignes, voire, dans un cas, de rien du tout: une nouvelle composée uniquement de son titre («C'est trop»). En tout, plus de quatre-vingts nouvelles en moins de cent cinquante pages. Gilles Pellerin aime jouer avec les formes, les clichés, les mots. On en trouve de joliment inventés dans l'une des nouvelles les plus drôles (il y en a plusieurs), intitulée "L'esprit et la lettre»: «Ma très chère, je ne sais pas si vous appragancerez ma démarche de ce matin. Le seul fait que j'opte pour le vouvoiement et une certaine solennité doit vous indiquer quel bramantier j'y parmane.» (54) Certaines nouvelles très courtes ont la force lapidaire d'un aphorisme, comme cet «Épitaphe»: «Il ne faisait jamais rien de bon; le reste, il le faisait mal.» Ailleurs, le trait passe à côté de la cible: «Fils, mari, gendre, ce n'était pas assez? Voilà que je suis père. Ignorant tout du scénario.» («L'esprit de famille») Le recueil saute allègrement d'un registre à l'autre, d'un style à l'autre, d'un lieu à l'autre. Il y en a pour tous les goûts. Cela ressemble tantôt à un petit poème en prose, tantôt à une satire politique ou à une critique mordante de quelque fait de société.

Ces changements apparents de style n'affaiblissent aucunement l'unité d'ensemble. Le style reste tributaire de la forme même de la nouvelle, ramenée ici à sa contrainte première, la brièveté. Chaque nouvelle obéit à un principe d'économie qui oblige l'écrivain à saisir instantanément l'attention du lecteur. Le curieux titre Ï (i tréma) ressemble à une note de musique stridente, ce qui convient d'ailleurs assez bien à l'expression tendue des personnages mis en scène par Gilles Pellerin, surpris à un moment de leur existence. Quand il raconte quelque action, Pellerin n'y va pas par quatre chemins: il se met à écrire des phrases saccadées qui se mangent les unes les autres tant elles sont pressées d'arriver au but. L'une des plus belles réussites dans le genre est la nouvelle «Jamais chez elle», qui raconte les efforts désespérés

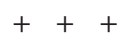

5 Gilles Pellerin, Nous aurions un petit genre: publier des nouvelles, Québec, L’Instant même, 1997. 
d'un homme pour téléphoner chez Diane à partir d'un café où l'on vend des jetons à prix fort. Quand il décrit des lieux (il adore décrire la ville de Québec) ou quand il tente (avec un succès moindre) d'entrer dans le monde affectif de ses personnages, il se laisse aller à une sorte de rêverie un peu vague et ne craint pas d'allonger la phrase, comme dans «L'entrée en Mauricie»:

En un endroit indécis entre Grondines et La Pérade, qu'on pourrait croire aplati par l'insignifiance, plaine jaune traversée par l'autoroute, je devine dans le lointain, dans le couchant, le panneau souhaitant la bienvenue dans la région touristique du Cœur-du-Québec, comme si, sur cette frontière, je pouvais me dire que je rentre en Mauricie. (25)

Ailleurs, le nouvelliste semble pasticher les plus mauvais poètes: «L'entrelacs sans fin des autoroutes. La nuit vacillante sous la bruine intermittente. Çà et là des masses surgies du ciel ou d’entre les pilotis de béton.» (46) («Prochaine sortie à droite») Au total, cependant, le recueil fait sourire plus qu'il n'émeut. Il a quelque chose de l'exercice de style, de la prouesse, presque de la gageure. Il relève d'une indéniable maîtrise technique, d'un métier. Mais la littérature est-elle vraiment un métier?

La question n'est pas sans rapport avec les transformations opérées depuis que la création littéraire est devenue une matière à enseignement comme une autre. En raison de sa brièveté, la nouvelle se prête particulièrement bien au format des ateliers d'écriture. Cela produit d'incontestables talents, des nouvellistes qui comprennent bien les règles du genre et qui en jouent avec confiance et ingéniosité. Jamais peut-être n'a-t-on vu autant d'écrivains ayant une telle virtuosité technique. Sans doute faut-il se féliciter d'un tel succès. Et pourtant, on cherche en vain la grande émotion, celle qui demeure après qu'on a rangé le livre dans la bibliothèque. Rien à voir avec les «coups de cœur» dont les libraires se servent pour nous assommer chaque semaine. Des livres où le métier serait là, mais invisible, au service d'autre chose qui ne s'enseigne peut-être pas et qui fait toute la grandeur de l'écrivain. 